\title{
Coverings, heat kernels and spanning trees
}

\author{
Fan Chung ${ }^{\dagger} *$ \\ University of Pennsylvania \\ Philadelphia, Pennsylvania 19104 \\ chung@hans.math.upenn.edu \\ S.-T. Yau ${ }^{\dagger}$ \\ Harvard University \\ Cambridge, Massachusetts 02138 \\ yau@math.harvard.edu
}

Submitted: May 1, 1998; Accepted: December 12, 1998.

AMS Subject Classification: 05C50, 05Exx, 35P05, 58G99.

\begin{abstract}
We consider a graph $G$ and a covering $\tilde{G}$ of $G$ and we study the relations of their eigenvalues and heat kernels. We evaluate the heat kernel for an infinite $k$-regular tree and we examine the heat kernels for general $k$-regular graphs. In particular, we show that a $k$-regular graph on $n$ vertices has at most

$$
(1+o(1)) \frac{2 \log n}{k n \log k}\left(\frac{(k-1)^{k-1}}{\left(k^{2}-2 k\right)^{k / 2-1}}\right)^{n}
$$

spanning trees, which is best possible within a constant factor.
\end{abstract}

\section{Introduction}

We consider a weighted undirected graph $G$ (possibly with loops) which has a vertex set $V=V(G)$ and a weight function $w: V \times V \rightarrow \mathbb{R}$ satisfying

$$
w(u, v)=w(v, u) \text { and } w(u, v) \geq 0 .
$$

*Research supported in part by NSF Grant No. DMS 98-01446

${ }^{\dagger}$ Research supported in part by NSF Grant No. DMS 95-04834 
If $w(u, v)>0$, then we say $\{u, v\}$ is an edge and $u$ is adjacent to $v$. A simple graph is the special case where all the weights are 0 or 1 and $w(v, v)=0$ for all $v$. In this paper, by a graph we mean a weighted graph unless specified.

The degree $d_{v}$ of a vertex $v$ is defined to be:

$$
d_{v}=\sum_{u} w(u, v)
$$

A graph is regular if all its degrees are the same. For a vertex $v$ in $G$, the neighborhood $N(v)$ of $v$ consists of all vertices adjacent to $v$.

This paper is organized as follows: In Section 2, we define a covering of a graph and give several examples. In Section 3, we give the definitions for the Laplacian, eigenvalues and the heat kernel of a graph. In Section 4, we consider the relations between the eigenvalues of a graph and the eigenvalues of its covering. In particular, we give a proof for determining the eigenvalues and their multiplicities of a strongly cover-regular graph $G$ from the eigenfunctions of the (smaller) graph covered by $G$. In Section 5 , we derive the heat kernel of an infinite $k$-regular tree. Then in Section 6 , we consider heat kernels of some $k$-regular graphs. In Section 7 , we consider the relations between the trace of the heat kernel and the number of spanning trees in a graph. In Section 8, we focus on an old problem of determining the maximum number of spanning trees in a $k$-regular graph. We consider the zeta function of a graph and we improve the upper and lower bounds for the maximum number of spanning trees in a $k$-regular graph on $n$ vertices.

\section{The coverings of graphs}

Suppose we have two graphs $\tilde{G}$ and $G$. We say $\tilde{G}$ is a covering of $G$ (or $G$ is covered by $\tilde{G}$ ) if there is a mapping $\pi: V(\tilde{G}) \rightarrow V(G)$ satisfying the following two properties:

(i) There is an $m \in \mathbb{R}^{+} \cup\{\infty\}$, called the index of $\pi$, such that for $u, v \in V(G)$, we have

$$
\sum_{\substack{x \in \pi^{-1}(u) \\ y \in \pi^{-1}(v)}} w(x, y)=m w(u, v) .
$$

(ii) For $x, y \in V(\tilde{G})$ with $\pi(x)=\pi(y)$ and $v \in V(G)$, we have

$$
\sum_{z \in \pi^{-1}(v)} w(z, x)=\sum_{z^{\prime} \in \pi^{-1}(v)} w\left(z^{\prime}, y\right) .
$$

Remark 1: For simple graphs $G$ and $\tilde{G}$, (i) is equivalent to

(i') For every $\{u, v\} \in E(G)$, we have

$$
|\{\{x, y\} \in E(\tilde{G}): \pi(x)=u, \pi(y)=v\}|=m .
$$


And (ii) is equivalent to

(ii') For $x, y \in V(\tilde{G})$ with $\pi(x)=\pi(y)$, and $v$ adjacent to $\pi(x)$ in $G$, we have

$$
\left|N(x) \cap \pi^{-1}(v)\right|=\left|N(y) \cap \pi^{-1}(v)\right| .
$$

In other words, $\pi^{-1}$ defines a so-called equitable partition of $V(\tilde{G})$ which has been studied extensively in the literature. The reader is referred to Cvetković, Doob and Sachs [5], McKay [14], Godsil and McKay [12].

Example 1: Suppose $\tilde{G}=C_{2 n}$, the cycle on $2 n$ vertices and $G=P_{n+1}$, the path on $n+1$ vertices. The covering has index 2 since each edge of $P_{n+1}$ is covered by two edges of $C_{2 n}$.

Example 2: A graph $\tilde{G}$ is said to be a regular covering of $G$ if for a fixed vertex $v$ in $V(G)$ and for any vertex $x$ of $V(\tilde{G}), \tilde{G}$ is a covering of $G$ under a mapping $\pi_{x}$ which maps $x$ into $v$. In addition, if $\pi_{x}^{-1}$ is just $x$, we say $\tilde{G}$ is a strong regular covering of $G$. A graph $G$ is said to be distance regular if $G$ is a strong regular covering of a (weighted) path $P$ (with possible non-zero $w(v, v)$ ). For example, for a vertex $x$ in $V(G)$, we can consider a mapping $\pi_{x}$ so that all vertices $y$ at distance $i$ from $x$ are mapped to the $i$-th vertex of $P$. This definition is equivalent to the definition of distance regular graphs, given by Biggs [2].

Example 3: Let $T_{k}$ denote an infinite $k$-tree. It is not hard to check that $T_{k}$ is a covering of a $k$-regular graph $G$. More on this will be discussed in Sections 5 and 6 .

We note that in a covering $\tilde{G}$ of $G$, the vertices $v$ in $G$ can have preimages $\pi^{-1}(v)$ of different sizes (as in Example 2). In addition, the degrees of vertices in $\tilde{G}$ or $G$ are not necessarily the same. Nevertheless, there is a certain uniformity in the preimage of a vertex as illustrated in the following facts:

Fact 1 Suppose $\tilde{G}$ is a covering of $G$ under $\pi$ with index $m$. Then for $x \in \pi^{-1}(v)$, we have

$$
\left|\pi^{-1}(v)\right| \sum_{z \in \pi^{-1}(u)} w(z, x)=m w(u, v) .
$$

The proof follows from (i) and (ii). For a simple graph, Fact 1 implies

$$
\left|\pi^{-1}(v)\right| \cdot\left|N(x) \cap \pi^{-1}(u)\right|=m .
$$

As an immediate consequence, we have

Fact 2 Suppose $\tilde{G}$ is a covering of $G$ under $\pi$ with edge multiplicity $m$. Then for $x, y \in \pi^{-1}(v)$, we have

$$
d_{x}=d_{y}
$$


THE ElECtronic JOURnAL OF COMBINATORICS 6 (1999), \#R12

\section{The Laplacian and the heat kernel of a graph}

For a weighted graph $G$ on $n$ vertices associated with a weight function $w$, we consider the combinatorial Laplacian $L$ of $G$.

$$
L(u, v)= \begin{cases}d_{v}-w(v, v) & \text { if } u=v \\ -w(u, v) & \text { if } u \text { and } v \text { are adjacent } \\ 0 & \text { otherwise }\end{cases}
$$

In particular, for a function $f: V \rightarrow \mathbb{R}$, we have

$$
L f(v)=\sum_{y}(f(v)-f(u)) w(u, v) .
$$

Let $T$ denote the diagonal matrix with the $(v, v)$-th entry having value $d_{v}$. The (normalized) Laplacian of $G$ is defined to be

$$
\mathcal{L}(u, v)= \begin{cases}1-\frac{w(v, v)}{d_{v}} & \text { if } u=v, \text { and } d_{v} \neq 0 \\ -\frac{w(u, v)}{\sqrt{d_{u} d_{v}}} & \text { if } u \text { and } v \text { are adjacent } \\ 0 & \text { otherwise. }\end{cases}
$$

In other words, we have

$$
\mathcal{L}=T^{-1 / 2} L T^{-1 / 2}
$$

For a $k$-regular graph, we have

$$
\mathcal{L}=I-\frac{1}{k} A
$$

where $A$ is the adjacency matrix.

We denote the eigenvalues of $\mathcal{L}$ by $0=\lambda_{0} \leq \lambda_{1} \cdots \leq \lambda_{n-1}$ (which are sometimes called the eigenvalues of $G$ ). If $G$ is connected, we have $0<\lambda_{1}$. The reader is referred to [7] for various properties of eigenvalues of a graph.

In this paper, we mainly deal with connected graphs. Let $g$ denote an eigenfunction of $\mathcal{L}$ associated with eigenvalue $\lambda$. It is sometimes convenient to consider $f=T^{-1 / 2} g$, called the harmonic eigenfunction, which satisfies, for every vertex $v$ of G,

$$
\sum_{u}(f(v)-f(u)) w(u, v)=\lambda d_{v} f(v)
$$

For a graph $G$, we consider the heat kernel $h_{t}$, which is defined for $t \geq 0$ as follows: 


$$
\begin{aligned}
h_{t} & =\sum_{i} e^{-\lambda_{i} t} P_{i} \\
& =e^{-t \mathcal{L}} \\
& =I-t \mathcal{L}+\frac{t^{2}}{2} \mathcal{L}^{2}-\ldots
\end{aligned}
$$

where $P_{i}$ denotes the projection into the eigenspace associated with eigenvalue $\lambda_{i}$. In particular,

$$
h_{0}=I
$$

and $h_{t}$ satisfies the heat equation

$$
\frac{\partial h_{t}}{\partial t}=-\mathcal{L} h_{t}
$$

For any two vertices $x, y \in V$, we have

$$
h_{t}(x, y)=\sum_{i} e^{-\lambda_{i} t} \phi_{i}(x) \phi_{i}(y)
$$

where $\phi_{i}$ 's are orthonormal eigenfunctions of the Laplacian $\mathcal{L}$.

In particular, the trace of $h_{t}$ satisfies

$$
\begin{aligned}
\operatorname{Trh}_{t} & =\sum_{x} h_{t}(x, x) \\
& =\sum_{i} e^{-\lambda_{i} t} .
\end{aligned}
$$

\section{Eigenvalues of a graph and its covering}

If $\tilde{G}$ is a covering of $G$, their eigenvalues are intimately related. Namely, the spectrum of a large (covering) graph can often be determined from a small (covered) graph. This provides a simple method for determining the spectrum of certain families of graphs. Such approaches have long been studied in the literature. Here we will list several facts which will be used later. The proofs of some of these facts can be found in Godsil and McKay [12] (in which the definitions involve $(0,1)$ matrices but the proofs often can be adapted for general weighted graphs). We will sketch the proofs here for the sake of completeness.

If $\tilde{G}$ is a covering of $G$, we can "lift" the harmonic eigenfunction $f$ of $G$ to $\tilde{G}$ by defining, for each vertex $x$ in $\tilde{G}, f(x)=f(u)$ where $u=\pi(x)$. From definition (ii) of covering, we have

$$
\begin{aligned}
\sum_{y}(f(x)-f(y)) w(x, y) & =\sum_{v}(f(u)-f(v)) w(u, v) \\
& =\lambda d_{x}
\end{aligned}
$$

Therefore we have 
Lemma 1 If $\tilde{G}$ is a covering of $G$, then an eigenvalue of $G$ is an eigenvalue of $\tilde{G}$.

For each $x \in \pi^{-1}(v)$,

$$
\sum_{y}(f(x)-f(y)) w(x, y)=\lambda f(x) d_{x}
$$

By summing over $x$ in $\pi^{-1}(v)$, we have

$$
\sum_{x \in \pi^{-1}(v)} \sum_{y}(f(x)-f(y)) w(x, y)=\lambda \sum_{x \in \pi^{-1}(v)} f(x) d_{x} .
$$

We define the induced mapping of $f$ in $G$, denoted by $\pi f: V(G) \rightarrow \mathbb{R}$ by

$$
(\pi f)(v)=\sum_{x \in \pi^{-1}(v)} \frac{f(x) d_{x}}{d_{v}}
$$

Then, for $g=\pi f$, we have

$$
\sum_{u}(g(v)-g(u)) w(u, v)=\lambda g(v) d_{v}
$$

If $g$ is nontrivial, $\lambda$ is an eigenvalues of $\tilde{G}$. Thus we have shown the following:

Lemma 2 Suppose $\tilde{G}$ is a covering of $G$ and. If a harmonic eigenfunction $f$ of $\tilde{G}$, associated with an eigenvalue $\lambda$, has a nontrivial image in $G$, then $\lambda$ is also an eigenvalue for $G$.

Lemma 3 Suppose $\tilde{G}$ is a strong regular covering of $G$. Then, $\tilde{G}$ and $G$ have the same eigenvalues.

Proof: For any nontrivial harmonic eigenfunction $f$ of $\tilde{G}$ we can choose $v$ to be a vertex with nonzero value of $f$. The induced mapping of $f$ in $G$ has a nonzero value at $v$ and therefore is a nontrivial harmonic eigenfunction for $G$. From Lemma 2, we see that any eigenvalue of $\tilde{G}$ is an eigenvalue of $G$. By Lemma 1 , we conclude that $\tilde{G}$ and $G$ have the same eigenvalues.

Therefore the eigenvalues of a covering graph $\tilde{G}$ can be determined by computing the eigenvalues of a smaller graph $G$. However, the multiplicities for the eigenvalues in $\tilde{G}$ are, in general, different from those in $G$ since, for example, $\tilde{G}$ and $G$ can have different numbers of vertices. Nevertheless, the multiplicities of eigenvalues of $\tilde{G}$ and $G$ are related through the relations of their heat kernels.

Lemma 4 Suppose $\tilde{G}$ is a covering of $G$. Let $\tilde{h}_{t}$ and $h_{t}$ denote the heat kernels of $\tilde{G}$ and $G$, respectively. Then we have

$$
\sum_{x \in \pi^{-1}(u)} \sum_{y \in \pi^{-1}(v)} \tilde{h}_{t}(x, y)=\sqrt{\left|\pi^{-1}(u)\right| \cdot\left|\pi^{-1}(v)\right|} h_{t}(u, v) .
$$


Proof: We note that the heat kernel $h_{t}(u, v)$ satisfies

$$
h_{t}(u, v)=e^{-t} \sum_{r} S_{r}(u, v) \frac{t^{r}}{r !}
$$

where $S_{r}$ is the sum of weights of all walks of length $r$ joining $u$ and $v$. (Here a walk $p_{r}$ is a sequence of vertices $u_{0}, \ldots, u_{r}$ such that $u_{i}=u_{i+1}$ or $\left\{u_{i}, u_{i+1}\right\}$ is an edge. The weight of a walk is the product of $w\left(u_{i}, u_{i+1}\right) / \sqrt{d\left(u_{i}\right) d\left(u_{i+1}\right)}$, for $i=0, \ldots, r-1$.) We want to show that the total weights of the paths in $\tilde{G}$ lifted from $p_{r}$ (i.e., whose image in $G$ is $\left.p_{r}\right)$ is exactly the weight of $p_{r}$ in $G$ multiplied by $\sqrt{\left|\pi^{-1}\left(u_{0}\right)\right| \cdot\left|\pi^{-1}\left(u_{r}\right)\right|}$. Let $p_{r-1}$ denote the walk $u_{0}, \ldots, u_{r-1}$. Suppose $u_{r-1} \neq u_{r}$ (The other case is easy). For each path $\tilde{p}_{r-1}$ lifted from $p_{r-1}$, its extensions to paths lifted from $p_{r}$ has total weights

$$
\begin{aligned}
& w\left(\tilde{p}_{r-1}\right) \cdot \sum_{z \in \pi^{-1}\left(u_{r}\right)} \frac{-w\left(u_{r-1}, z\right)}{\sqrt{d\left(u_{r-1}\right) d(z)}} \\
= & w\left(\tilde{p}_{r-1}\right) \frac{-m w\left(u_{r-1}, u_{r}\right) /\left|\pi^{-1}\left(u_{r-1}\right)\right|}{\left.\sqrt{m d\left(u_{r-1}\right) m d\left(u_{r}\right) /\left(\left|\pi^{-1}\left(u_{r-1}\right)\right|\right.}\left|\pi^{-1}\left(u_{r}\right)\right|\right)} \\
= & w\left(\tilde{p}_{r-1}\right) \frac{-w\left(u_{r-1}, u_{r}\right)}{\sqrt{d\left(u_{r-1}\right) d\left(u_{r}\right)}} \sqrt{\frac{\left|\pi^{-1}\left(u_{r}\right)\right|}{\left|\pi^{-1}\left(u_{r-1}\right)\right|}} .
\end{aligned}
$$

By summing over all $\tilde{p}_{r-1}$, we have

$$
\sum_{x \in \pi^{-1}(u)} \sum_{y \in \pi^{-1}(v)} S_{r}(x, y)=\sqrt{\left|\pi^{-1}(u)\right| \cdot\left|\pi^{-1}(v)\right|} S_{r}(u, v) .
$$

Therefore, we complete the proof of Lemma 4.

As a consequence of Lemma 4, we have

Corollary 1 Suppose $\tilde{G}$ is a strong regular covering of $G$. Let $\tilde{h}_{t}$ and $h_{t}$ denote the heat kernels of $\tilde{G}$ and $G$, respectively. For $x \in \pi^{-1}(u)$, we have

$$
\sum_{y \in \pi^{-1}(v)} \tilde{h}_{t}(x, y)=\sqrt{\frac{\left|\pi^{-1}(v)\right|}{\left|\pi^{-1}(u)\right|}} h_{t}(u, v) .
$$

Corollary 2 Suppose $G$ is a distance regular graph which is a covering of a path $P$ with vertices $v_{0}, \ldots, v_{p}$ where $p=D(G)$. Suppose $G$ and $P$ have heat kernels $\tilde{h}_{t}$ and $h_{t}$, respectively. For any two vertices $x$ and $y$ in $G$ with distance $d(x, y)=r$, we have

$$
\tilde{h}_{t}(x, y)=\sqrt{\left|\pi^{-1}\left(u_{r}\right)\right|} h_{t}\left(v_{0}, v_{r}\right) .
$$


Theorem 1 Suppose $\tilde{G}$ is a strong regular covering of $G$. Let $v$ denote the vertex of $G$ with preimage in $\tilde{G}$ consisting of one vertex. Then any eigenvalue $\lambda$ of $\tilde{G}$ has multiplicity

$$
n \sum_{i} \frac{\phi_{i}^{2}(v)}{\left\|\phi_{i}\right\|^{2}},
$$

where $n=|V(\tilde{G})|$ and $\phi_{i}$ 's span the eigenspace of $\lambda$ in $G$. If the eigenvalue $\lambda$ has multiplicity 1 in $G$ with eigenfunction $\phi$, then the multiplicity of $\lambda$ in $\tilde{G}$ is

$$
\frac{n \phi^{2}(v)}{\|\phi\|^{2}} \text {. }
$$

Proof: $\quad$ Suppose $\tilde{G}$ has heat kernel $H_{t}$ and $G$ has heat kernel $h_{t}$. Since $\tilde{G}$ is a strong regular covering of $G$, we have

$$
\begin{aligned}
\operatorname{Tr}\left(\tilde{h}_{t}\right) & =\sum_{x \in V(\tilde{G})} H_{t}(x, x) \\
& =n h_{t}(v, v) \\
& =n \sum_{j} e^{-t \lambda_{j}} \frac{\phi_{j}^{2}(v)}{\left\|\phi_{j}\right\|^{2}} .
\end{aligned}
$$

Therefore, the multiplicity of $\lambda_{j}$ in $\tilde{G}$ is exactly

$$
\frac{n \phi_{j}^{2}(v)}{\left\|\phi_{j}\right\|^{2}}
$$

if the multiplicity of $\lambda$ in $G$ is 1 and. In general, the multiplicity of $\lambda$ in $\tilde{G}$ is

$$
n \sum_{i} \frac{\phi_{i}^{2}(v)}{\left\|\phi_{i}\right\|^{2}}
$$

where $\phi_{i}$ 's span the eigenspace of $\lambda$ in $G$.

As an immediate consequence of Theorem 1, we have the following:

Corollary 3 A distance regular graph $G$ with diameter $D$ has $D+1$ distinct eigenvalues $\lambda$ 's which are the eigenvalues of a weighted path $P$ of length $D$. (The weight of edge $\left\{v_{i}, v_{i+1}\right\}$ in $P$ is the number of edges joining a vertex at distance $i$ from $x$ to a vertex at distance $i+1$ from $x$ for a fixed number $x$. The weight of the loop $\left\{v_{i}, v_{i}\right\}$ is twice the number of edges with both endpoints at distance $i$ from $x$.) The multiplicity of $\lambda$ in $G$ is

$$
\frac{n \phi^{2}(x)}{\|\phi\|^{2}}
$$

where $n$ is the number of vertices in $G$ and $\phi$ is the eigenfunction of $\lambda$ of the Laplacian of $P$. 
Example 4: The Petersen graph $G$ is a covering for a path $P$ of 3 vertices. It is easy to check that $P$ has three eigenvalues $0,2 / 3,5 / 3$ with eigenfunctions $\phi_{0}=(\sqrt{3}, \sqrt{6}, \sqrt{18})$, $\phi_{1}=(\sqrt{3}, 1,-\sqrt{2})$ and $\phi_{2}=(\sqrt{6},-2 \sqrt{2}, 1)$, respectively. Using Lemma 8 , we see that eigenvalues $0,2 / 3,5 / 3$ have multiplicities $1,5,4$ in $G$, respectively.

\section{The heat kernel of $k$-trees}

Let $T_{k}$ (or $k$-tree, in short) denote an infinite $k$-regular tree. Let $T_{k, l}$ denote an $l$-level tree with a root at the 0 -th level. The $l$-th level consists of the $k(k-1)^{l-1}$ vertices at distance $l$ from the root. The infinite tree can be viewed as taking the limit of $T_{k, l}$ as $l$ approaches infinity.

The heat kernel of $T_{k}$ plays a central role in examining the spectrum of any $\mathrm{k}$ regular graph. To determine the heat kernel of $T_{k}$, we can use the covering theorem in the previous section. The study of eigenvalues and eigenfunctions of $T_{k}$ can be found in many papers in the literature $[1,3,9,17,19]$. Here we will give a self-contained proof for establishing the explicit formula for the kernel of the $k$-tree, for $k \geq 3$. For the case of $k=2, T_{2}$ is just the infinite path. This special case and its cartesian products were examined in [6].

$T_{k}$ can be regarded as a covering of the following weighted path $P$. The vertex of $P$ is $\{0,1,2, \ldots\}$. For $j>0$, the edge joining $j-1$ to $j$ has weight $k(k-1)^{j-1}$. the covering mapping $\pi$ is defined by assigning all vertices in the $j$-th level to vertex $j$ in $P$. The Laplacian $\mathcal{L}$ for the weighted path has entries

$$
\mathcal{L}(i, j)=\left\{\begin{array}{cl}
1 & \text { if } i=j \\
-\frac{1}{\sqrt{k}} & \text { if }(i, j)=(0,1) \text { or }(1,0) \\
-\frac{\sqrt{k-1}}{k} & \text { if }|i-j|=1, i, j \neq 0 \\
0 & \text { otherwise }
\end{array}\right.
$$

We observe that $\mathcal{L}$ is quite close to $I-\frac{\sqrt{k-1}}{k} M$ where $M$ is the cyclic operator with $M(i, i+1)=M(i+1, i)=\frac{\sqrt{k-1}}{k}$ for $i \geq 0$ and 0 , otherwise. Intuitively, the eigenvalues of $T_{k}$ are just, for a fixed integer $l$,

$$
1-\frac{2 \sqrt{k-1}}{k} \cos \frac{\pi j}{l} \text { for } j=1, \ldots, l-1
$$

in addition to the eigenvalues 0 and 2 .

In order to examine the eigenvalues and eigenfunctions of $P$ explicitly, we consider 
the following $l \times l$ matrix $\mathcal{L}^{(l)}$, for $l \geq 3$ :

$$
\mathcal{L}^{(l)}=\left(\begin{array}{cccccc}
1 & -\frac{1}{\sqrt{k}} & 0 & \cdots & \cdots & 0 \\
-\frac{1}{\sqrt{k}} & 1 & -\frac{\sqrt{k-1}}{k} & 0 & \cdots & 0 \\
0 & -\frac{\sqrt{k-1}}{k} & 1 & -\frac{\sqrt{k-1}}{k} & \ldots & 0 \\
\cdots & \cdots & \cdots & \cdots & -\frac{\sqrt{k-1}}{k} & 0 \\
0 & \cdots & \cdots & \cdots & 1 & -\sqrt{\frac{k-1}{k}} \\
0 & \cdots & \cdots & \cdots & -\sqrt{\frac{k-1}{k}} & 1
\end{array}\right)
$$

where

$$
\mathcal{L}^{(l)}(i, j)=\left\{\begin{array}{cl}
1 & \text { if } i=j, \\
-\frac{1}{\sqrt{k}} & \text { if }(i, j)=(0,1) \text { or }(1,0), \\
-\frac{\sqrt{k-1}}{\sqrt{k}} & \text { if }|i-j|=1,0<i, j<l, \\
-\sqrt{\frac{k-1}{k}} & \text { if }(i, j)=(l-1, l) \text { or }(l, l-1), \\
0 & \text { otherwise. }
\end{array}\right.
$$

The eigenvalues of $\mathcal{L}^{(l)}$ are 0,2 and

$$
1-\frac{2 \sqrt{k-1}}{k} \cos \frac{\pi j}{l} \text { for } j=1, \ldots, l-1 .
$$

The eigenfunction $\phi_{0}$ associated with eigenvalue 0 is $\phi_{0}=f_{0} /\left\|f_{0}\right\|$ where $f_{0}$ is defined as follows:

$$
\begin{aligned}
f_{0}(0) & =1, \\
f_{0}(p) & =\sqrt{k(k-1)^{p-1}}, \\
f_{0}(l) & =\sqrt{(k-1)^{l-1}} .
\end{aligned} \quad \text { for } 1 \leq p \leq l-1,
$$

The eigenfunction $\phi_{l}$ associated with eigenvalue 2 is $\phi_{l}=f_{l} /\left\|f_{l}\right\|$ where $f_{l}$ is defined as follows:

$$
\begin{aligned}
& f_{l}(0)=1 \\
& f_{l}(p)=(-1)^{p} \sqrt{k(k-1)^{p-1}}, \\
& f_{l}(l)=(-1)^{l} \sqrt{(k-1)^{l-1}}
\end{aligned}
$$

The eigenfunction $\phi_{j}$, for $j=1, \cdots, l-1$, associated with eigenvalue $1-\frac{2 \sqrt{k-1}}{k} \cos \frac{\pi j}{l}$ is $f_{j} /\left\|f_{j}\right\|$ where

$$
\begin{array}{rlrl}
f_{j}(0) & =\sqrt{\frac{k}{k-1}} \sin \frac{\pi j}{l}, & \\
f_{j}(p) & =\sin \frac{\pi j(p+1)}{l}-\frac{1}{k-1} \sin \frac{\pi j(p-1)}{l}, & \text { for } 1 \leq p \leq l-1, \\
f_{j}(l) & =-\frac{\sqrt{k}}{k-1} \sin \frac{\pi j}{l} .
\end{array}
$$


It is easy to compute, for $j=1, \cdots, l-1$,

$$
\left\|f_{j}\right\|^{2}=\frac{l k^{2}}{2(k-1)^{2}}\left(1-\frac{4(k-1)}{k^{2}} \cos ^{2} \frac{\pi j}{l}\right) .
$$

Therefore the heat kernel $h^{(l)}$ of $P^{(l)}$ satisfies

$$
h^{(l)}(0,0)=\sum_{j=1}^{l-1} \frac{e^{-t\left(1-\frac{2 \sqrt{k-1}}{k} \cos \frac{\pi j}{l}\right)} \sin ^{2} \frac{\pi j}{l}}{\frac{l k}{2(k-1)}\left(1-\frac{4(k-1)}{k^{2}} \cos ^{2} \frac{\pi j}{l}\right)}+\frac{1}{\left\|f_{0}\right\|^{2}}+\frac{1}{\left\|f_{l}\right\|^{2}} .
$$

When $l$ approaches infinity, the heat kernel $h$ of $P$ satisfies:

$$
h_{t}(0,0)=\frac{2 k(k-1)}{\pi} \int_{0}^{\pi} \frac{e^{-t\left(1-\frac{2 \sqrt{k-1}}{k} \cos x\right)} \sin ^{2} x}{k^{2}-4(k-1) \cos ^{2} x} d x .
$$

In general, for $a \geq 1$, we have

$h_{t}(0, a)=\frac{2 \sqrt{k(k-1)}}{\pi} \int_{0}^{\pi} \frac{e^{-t\left(1-\frac{2 \sqrt{k-1}}{k} \cos x\right)} \sin x[(k-1) \sin (a+1) x-\sin (a-1) x]}{k^{2}-4(k-1) \cos ^{2} x} d x$.

For the infinite $k$-tree $T_{k}$, its heat kernel is denoted by $H_{t}$. For two vertices $x, y$ in $T_{k}$, we will write $H_{t}(x, y)=H_{t}(0, d(x, y))$ where $d(x, y)$ denotes the distance of $x$ and $y$ in $T_{k}$. In particular, $H_{t}(x, x)=H_{t}(0,0)$ for all vertices $x$. Using Lemma 4 and the fact that the infinite $k$-tree is a covering of $P$, we have the following:

Theorem 2 The heat kernel $H_{t}$ of the infinite $k$-tree satisfies

$$
\begin{aligned}
& H_{t}(0,0)=\frac{2 k(k-1)}{\pi} \int_{0}^{\pi} \frac{e^{-t\left(1-\frac{2 \sqrt{k-1}}{k} \cos x\right)} \sin ^{2} x}{k^{2}-4(k-1) \cos ^{2} x} d x \\
& H_{t}(0, a)=\frac{2}{\pi(k-1)^{a / 2-1}} \int_{0}^{\pi} \frac{e^{-t\left(1-\frac{2 \sqrt{k-1}}{k} \cos x\right)} \sin x[(k-1) \sin (a+1) x-\sin (a-1) x]}{k^{2}-4(k-1) \cos ^{2} x} d x .
\end{aligned}
$$

Corollary 4 The heat kernel $H_{t}(0,0)$ of the infinite $k$-tree can be written as

$$
\begin{aligned}
H_{t}(0,0) & =e^{-t} \sum_{r \geq 0} \sum_{j=0}^{r}\left(\begin{array}{c}
2 r \\
j
\end{array}\right) \frac{2 r-2 j+1}{2 r-j+1}(k-1)^{j}\left(\frac{t}{k}\right)^{2 r} \\
& =\frac{2(k-1)}{k} \sum_{s \geq 0}\left(\frac{4(k-1)}{k^{2}}\right)^{2 s} \frac{(2 s-1) ! !}{(2 s+2) ! !} \sum_{0 \leq j \leq s} \frac{t^{2} j}{(2 j) !}
\end{aligned}
$$

where $m$ !! denotes the product of all numbers less than or equal to $m$ and having the same parity as $m$.

We note that the first sum in the corollary above appeared in [15]. We remark that the heat kernel $H_{t}$ of the $k$-tree can be viewed as a basic building block for the heat kernel of any $k$-regular graph, which in turn is closely related to many major invariants of the graph. 
THE ELECTRONiC JoURnal of COMBInAtorics 6 (1999), \#R12

\section{The heat kernel of the $k$-tree and the heat kernel of a $k$-regular graph}

For a $k$-regular graph $G$, there is a natural mapping $\pi$ from $T_{k}$ to $G$ so that for each vertex $x$ in $T_{k}$, the neighbors of $x$ are mapped to neighbors of $\pi(x)$ in $G$ in an oneto-one fashion. Let $H_{t}$ denote the heat kernel of $T_{k}$. We here abuse the notation by writing $H_{t}(x, y)=H_{t}(0, d(x, y))$ for two vertices $x$ and $y$ at distance $d(x, y)$ in $T_{k}$.

Lemma 5 For a k-regular graph $G$, there is a covering $\pi$ from $T_{k}$ to $G$ and the heat kernel $h_{t}$ of $G$ satisfies

$$
h_{t}(u, v)=\sum_{y \in \pi^{-1}(u)} H_{t}(0, d(x, y))
$$

where $v=\pi(x), d(x, y)$ denotes the distance between $x$ and $y$ in $T_{k}$ and $H_{t}$ denotes the heat kernel of $T_{k}$.

In a graph $G$, a walk of length $s$ is a sequence of vertices $\left(v_{0}, v_{1}, \cdots, v_{s}\right)$ where $\left\{v_{i}, v_{i+1}\right\}$ is an edge for $i=0, \cdots, s-1$. If $v_{0}=v_{s}$, it is called a closed walk rooted at $v_{0}$. A walk $\left(v_{0}, v_{1}, \cdots, v_{s}\right)$ is said to be irreducible if $v_{j} \neq v_{j+2}$ for $j=0, \cdots, s-2$. If $v_{j}=v_{j+2}$ for some $j$, we can reduce the walk by deleting $v_{j}$ and $v_{j+1}$. A walk is said to be totally reducible if it can be reduced to a trivial walk of length 0 . Let $r_{j}$ denote the number of totally reducible walk rooted at any vertex. In McKay $[15,16], r_{j}$ 's have been extensively examined. From the definition of the heat kernel, we have the following:

Lemma 6 In a k-regular graph, the number $r_{s}$ of totally reducible walks of length $s$ rooted at any vertex satisfies

$$
H_{t}(0,0)=e^{-t} \sum_{j \geq 0} r_{j} \frac{(t / k)^{j}}{j !}
$$

where $H_{t}$ is the heat kernel of the infinite tree $T_{k}$.

Proof: We observe that $r_{j}$ is exactly the number of rooted closed walks of length $j$ in the infinite tree $T_{k}$. From the definition of $H_{t}$ we have

$$
\begin{aligned}
H_{t} & =e^{-t} \cdot e^{A / k} \\
& =e^{-t}\left(I+A \frac{t}{k}+A^{2} \frac{(t / k)^{2}}{2 !}+\cdots\right)
\end{aligned}
$$

where $A$ denotes the adjacency operator. Lemma 6 then follows. 
THE ELECTRONiC JOURNAL of COMBinatorics 6 (1999), \#R12

Lemma 7 For odd $j, r_{j}$ is zero and for the even case, we have

$$
\begin{aligned}
r_{2 j} & =\frac{4^{j+1} k(k-1)^{j+1}}{\pi} \int_{0}^{\pi / 2} \frac{\sin ^{2} x \cos ^{2 j} x}{k^{2}-4(k-1) \cos ^{2} x} d x \\
& \leq \frac{4^{j} k(k-1)^{j+1}}{2 j \sqrt{\pi j}(k-2)^{2}} .
\end{aligned}
$$

Proof: The proof follows from Lemma 5 and Lemma 6 which imply:

$$
\begin{aligned}
r_{2 j} & =\frac{\partial^{2 j}}{\partial t^{2 j}}\left(e^{t} H_{t}(0,0)\right) \\
& =\frac{4^{j+1} k(k-1)^{j+1}}{\pi} \int_{0}^{\pi / 2} \frac{\sin ^{2} x \cos ^{2 j} x}{k^{2}-4(k-1) \cos ^{2} x} d x .
\end{aligned}
$$

Therefore we have

$$
\begin{aligned}
r_{2 j} & \leq \frac{4^{j+1} k(k-1)^{j+1}}{\pi(k-2)^{2}} \int_{0}^{\pi / 2} \sin ^{2} x \cos ^{2 j} x d x \\
& =\frac{4^{j+1} k(k-1)^{j+1}}{\pi(k-2)^{2}(2 j+1)} \int_{0}^{\pi / 2} \cos ^{2 j+2} x d x \\
& =\frac{4^{j+1} k(k-1)^{j+1}}{\pi(k-2)^{2}(2 j+1)} \frac{2 j+1}{2 j+2} \frac{2 j-1}{2 j} \cdots \frac{1}{2} \frac{\pi}{4} \\
& \leq \frac{4^{j+1} k(k-1)^{j+1}}{\pi(k-2)^{2}} \frac{\sqrt{\pi}}{8(j+1) \sqrt{j}} \\
& =\frac{4^{j} k(k-1)^{j+1}}{2(j+1) \sqrt{\pi j}(k-2)^{2}} .
\end{aligned}
$$

We note that a similar upper bound was given in [16] as an asymptotic estimate for $r_{2 j}$.

Lemma 8 For a k-regular graph $G$, there is a covering $\pi$ from $T_{k}$ to $G$ and the heat kernel $h_{t}(u, v)$ of $G$ satisfies

$$
h_{t}(u, v)=\sum_{a=0}^{\infty} c_{a} H_{t}(0, a)
$$

where $c_{a}$ denotes the number of irreducible walks from $v$ to $u$ of length a.

\section{$7 \quad$ Spanning trees in a $k$-regular graph}

For a connected graph $G$, we consider the $\zeta$-function

$$
\zeta(s)=\sum_{i \neq 0} \frac{1}{\lambda_{i}^{s}}
$$


where $\lambda_{i}$ ranges over all nonzero eigenvalues of $G$.

It can be easily checked that

$$
-\zeta^{\prime}(0)=\sum_{i \neq 0} \log \lambda_{i}=\log \prod_{i \neq 0} \lambda_{i}
$$

where log denotes the natural logarithm.

Theorem 3 For a connected graph $G$, the number $\tau(G)$ of spanning trees in $G$ is equal to

$$
\frac{\prod_{x} d_{x}}{\sum_{x} d_{x}} e^{-\zeta^{\prime}(0)}
$$

where $d_{x}$ denotes the degree of $x$.

Proof: Suppose we consider the characteristic polynomial $p(x)$ of the Laplacian $\mathcal{L}$.

$$
p(x)=\operatorname{det}(\mathcal{L}-x I) .
$$

The coefficient of the linear term is exactly

$$
-\prod_{i \neq 0} \lambda_{i}
$$

On the other hand,

$$
p(x)=\operatorname{det} T^{-1} \operatorname{det}(L-x T)=\left(\prod_{x} d_{x}\right)^{-1} p_{1}(x) .
$$

By the well known matrix-tree theorem, the coefficient of the linear term of $p_{1}(x)$ is exactly $-\sum_{x} d_{x}$ times the number of spanning trees of $G$.

Thus, the number of spanning trees of a $k$-regular graph on $n$ vertices satisfies

$$
\tau(G)=\frac{k^{n-1}}{n} e^{-\zeta^{\prime}(0)}
$$

In the rest of the paper, we assume that $G$ is $k$-regular.

The trace function $\operatorname{Tr} h_{t}$ of $G$ satisfies

$$
\operatorname{Tr} h_{t}=\sum_{i} e^{-t \lambda_{i}}
$$

Therefore the zeta function satisfies

$$
\zeta(s)=\frac{1}{\Gamma(s)} \int_{0}^{\infty} t^{s-1}\left(\operatorname{Tr} h_{t}-1\right) d t
$$

by using the fact that

$$
\frac{1}{\Gamma(z)} \int_{0}^{\infty} e^{-\rho t} t^{z-1} d t=\frac{1}{\rho^{z}}
$$


THE ELECTRONiC JOURNAL OF COMBINATORICS 6 (1999), \#R12

\section{The maximum number of spanning trees in $k$ - regular graphs}

McKay [16] gave the following bounds for the maximum number of spanning trees over all $k$-regular graphs $G_{n}$ on $n$ vertices:

$$
c_{1} \frac{1}{n} C^{n} \leq \max \tau(G) \leq c_{2} \frac{\log n}{n} C^{n}
$$

where

$$
C=\frac{(k-1)^{k-1}}{\left(k^{2}-2 k\right)^{k / 2-1}}
$$

and $c_{1}$ and $c_{2}$ depend only on $k$ (in some complicated formula). He conjectured that the upper bound is the right order for $\max \tau\left(G_{n}\right)$. Here we will simplify the upper bound and prove that indeed it is best possible within a constant factor.

Theorem 4 For $k \geq 3$, the number $\tau\left(G_{n}\right)$ of spanning trees in a $k$-regular graph $G_{n}$ on $n$ vertices satisfies

$$
\tau\left(G_{n}\right) \leq(1+o(1)) \frac{2 \log n}{k n \log k}\left(\frac{(k-1)^{k-1}}{\left(k^{2}-2 k\right)^{k / 2-1}}\right)^{n}
$$

Theorem 5 For $k \geq 8$, there are $k$-regular graphs $G$ on $n$ vertices having the number $\tau\left(G_{n}\right)$ of spanning trees satisfying

$$
\tau(G) \geq(1+o(1)) \frac{\log n}{k n \log k}\left(\frac{(k-1)^{k-1}}{\left(k^{2}-2 k\right)^{k / 2-1}}\right)^{n} .
$$

We first need to establish the relation between the heat kernels $h_{t}$ and $H_{t}$. Let $r_{j}^{\prime}$ denote the total number of rooted closed walks of length $j$ which are not totally reducible. We then have

$$
\begin{aligned}
\operatorname{Tr} h_{t} & =e^{-t} \sum_{j \geq 0}\left(n r_{j}+r_{j}^{\prime}\right) \frac{(t / k)^{j}}{j !} \\
& =n H_{t}(0,0)+e^{-t} \sum_{j \geq 0} r_{j}^{\prime} \frac{(t / k)^{j}}{j !} .
\end{aligned}
$$

From equation (3), we have

$$
\zeta(s)=: \zeta_{0}(s)+\zeta_{1}(s)
$$

where

$$
\zeta_{0}(s)=\frac{n}{\Gamma(s)} \int_{0}^{\infty} t^{s-1} H_{t}(0,0) d t
$$


THE ELECTRONiC JOURNAL of COMBinatorics 6 (1999), \#R12

and

$$
\zeta_{1}(s)=\frac{1}{\Gamma(s)} \int_{0}^{\infty} t^{s-1} e^{-t}\left(\sum_{j \geq 0} r_{j}^{\prime} \frac{(t / k)^{j}}{j !}-e^{t}\right)
$$

We have

$$
\begin{aligned}
\zeta_{0}(s) & =\frac{n}{\Gamma(s)} \int_{0}^{\infty} t^{s-1} H_{t}(0,0) d t \\
& =\frac{2 n k(k-1)}{\pi \Gamma(s)} \int_{0}^{\infty} t^{s-1} \int_{0}^{\pi} \frac{e^{-t\left(1-\frac{2 \sqrt{k-1}}{k} \cos x\right)} \sin ^{2} x}{k^{2}-4(k-1) \cos ^{2} x} d x d t \\
& =\frac{2 n k(k-1)}{\pi} \int_{0}^{\pi} \frac{1}{\left(1-\frac{2 \sqrt{k-1}}{k} \cos x\right)^{s}} \cdot \frac{\sin ^{2} x}{k^{2}-4(k-1) \cos ^{2} x} d x .
\end{aligned}
$$

Therefore

$$
\begin{aligned}
\zeta_{0}^{\prime}(0) & =-\frac{2 n k(k-1)}{\pi} \int_{0}^{\pi} \frac{\sin ^{2} x}{k^{2}-4(k-1) \cos ^{2} x} \log \left(1-\frac{2 \sqrt{k-1}}{k} \cos x\right) d x \\
& =n \log \frac{k^{k / 2}(k-2)^{k / 2-1}}{(k-1)^{k-1}} .
\end{aligned}
$$

The above integral is evaluated by using the following formula given in [16]:

$$
\frac{k}{2 \pi} \int_{-\omega}^{\omega} \frac{\left(\omega^{2}-x^{2}\right)^{1 / 2}}{k^{2}-x^{2}} \log (1-\gamma x) d x=-\log \left(\eta\left(\frac{k-\eta}{k-1}\right)^{k / 2-1}\right)
$$

where $|\gamma|=1 / k<1 / \omega, \omega=2 \sqrt{k-1}$ and $\eta=\frac{1-\left(1-4(k-1) \gamma^{2}\right)^{1 / 2}}{2(k-1) \gamma^{2}}$.

It remains to evaluate $\zeta_{1}^{\prime}(0)$. We note that

$$
\begin{aligned}
n r_{j}+r_{j}^{\prime} & =\operatorname{Tr} A^{j} \\
& =k^{j} \sum_{i}\left(1-\lambda_{i}\right)^{j} .
\end{aligned}
$$

So, we have, for odd $\mathrm{j}$,

$$
\frac{r_{j}^{\prime}}{k^{j}} \geq 1+\sum_{i \neq 0}\left(1-\lambda_{i}\right)^{j}
$$

For the even case,

$$
\frac{r_{2 j}^{\prime}}{k^{2 j}} \geq 1+\sum_{i \neq 0}\left(1-\lambda_{i}\right)^{2 j}-\frac{n 4^{j} k(k-1)^{j+1}}{j \sqrt{\pi j}(k-2)^{2}} .
$$


For a fixed value $\beta$ (which will be chosen later), we have

$$
\begin{aligned}
\zeta_{1}(s)= & \frac{1}{\Gamma(s)} \int_{0}^{\infty} t^{s-1} e^{-t}\left(\sum_{j \geq 0} r_{j}^{\prime} \frac{(t / k)^{j}}{j !}-e^{t}\right) d t \\
\geq & \frac{1}{\Gamma(s)} \int_{0}^{\infty} t^{s-1} e^{-t}\left(-\sum_{j=0}^{2 \beta-1} \frac{(t / k)^{j}}{j !}\right) d t \\
& +\frac{1}{\Gamma(s)} \int_{0}^{\infty} t^{s-1} e^{-t}\left(\sum_{j>2 \beta}\left(r_{j}^{\prime} \frac{(t / k)^{j}}{j !}-\frac{(t / k)^{j}}{j !}\right)\right) d t .
\end{aligned}
$$

We note that for $j \geq 1$, and

$$
\rho(s)=\frac{1}{\Gamma(s)} \int_{0}^{\infty} t^{s-1} e^{-t}\left(\frac{t^{j}}{j !}\right) d t
$$

we have

$$
\rho^{\prime}(0)=\frac{1}{j} .
$$

Therefore

$$
\begin{aligned}
\zeta_{1}^{\prime}(0) & \geq-\sum_{j=1}^{2 \beta-1} \frac{1}{j}+\zeta_{2}^{\prime}(0) \\
& \geq-\log (2 \beta)+\zeta_{2}^{\prime}(0)
\end{aligned}
$$

where we define

$$
\zeta_{2}(s)=\frac{1}{\Gamma(s)} \int_{0}^{\infty} t^{s-1} e^{-t}\left(\sum_{j \geq 2 \beta}\left(r_{j}^{\prime} \frac{(t / k)^{j}}{j !}-\frac{(t / k)^{j}}{j !}\right)\right) .
$$

Here, we have

$$
\zeta_{2}(s)=\frac{1}{\Gamma(s)} \int_{0}^{\infty} t^{s-1} e^{-t}\left(\sum_{j \geq 2 \beta} \frac{t^{j}}{j !}\left(\sum_{i \neq 0}\left(1-\lambda_{i}\right)^{j}-\sum_{j \geq \beta} \frac{n 4^{j} k(k-1)^{j+1}}{j \sqrt{\pi j}(k-2)^{2} k^{2 j}}\right)\right) .
$$

By using equation (7) and inequality (8), we have

$$
\begin{aligned}
\zeta_{2}^{\prime}(0) & \geq \sum_{j \geq 2 \beta} \sum_{i \neq 0}\left(1-\lambda_{i}\right)^{j} \frac{1}{j}-\sum_{j \geq \beta} \frac{n 4^{j} k(k-1)^{j+1}}{j^{2} \sqrt{\pi j}(k-2)^{2} k^{2 j}} \\
& \geq-\sum_{j \geq \beta} \frac{n 4^{j} k(k-1)^{j+1}}{j^{2} \sqrt{\pi j}(k-2)^{2} k^{2 j}} \\
& \geq-2 \frac{n 4^{\beta} k(k-1)^{\beta+1}}{\beta^{2} \sqrt{\pi \beta}(k-2)^{2} k^{2 \beta}}
\end{aligned}
$$


by using $\lambda_{i} \leq 2$ and the fact that $\sum_{j \geq 2 \beta}\left(1-\lambda_{i}\right)^{j} / j \geq 0$.

Now, we are ready to prove Theorem 4 and 5 .

Proof of Theorem 4:

From (2) and (5), we have

$$
\begin{aligned}
\tau(G) & =\frac{k^{n-1}}{n} e^{-\zeta_{0}^{\prime}(0)-\zeta_{1}^{\prime}(0)} \\
& =\frac{k^{n-1}}{n}\left(\frac{(k-1)^{k-1}}{k^{k / 2}(k-2)^{k / 2-1}}\right)^{n} e^{-\zeta_{1}^{\prime}(0)} \\
& =\frac{1}{k n}\left(\frac{(k-1)^{k-1}}{\left(k^{2}-2 k\right)^{k / 2-1}}\right)^{n} e^{-\zeta_{1}^{\prime}(0)}
\end{aligned}
$$

By using the preceding lower bounds of $\zeta_{1}^{\prime}$ in (8), we have

$$
\tau(G) \leq \frac{2 \beta}{k n}\left(\frac{(k-1)^{k-1}}{\left(k^{2}-2 k\right)^{k / 2-1}}\right)^{n} e^{-\zeta_{2}^{\prime}(0)}
$$

We now choose $\beta$ as:

$$
\beta=\left\lceil\frac{\log n}{\log \frac{k^{2}}{4(k-1)}}\right\rceil .
$$

From (9), we have

$$
\begin{aligned}
\zeta_{2}^{\prime}(0) & \geq-2 \frac{n 4^{\beta} k(k-1)^{\beta+1}}{\beta^{2} \sqrt{\pi \beta}(k-2)^{2} k^{2 \beta}} \\
& \geq-2 \frac{k(k-1)}{\beta^{2} \sqrt{\pi \beta}(k-2)^{2}} .
\end{aligned}
$$

Therefore, we have

$$
\begin{aligned}
\tau(G) & \leq \frac{2 \beta}{k n}\left(\frac{(k-1)^{k-1}}{\left(k^{2}-2 k\right)^{k / 2-1}}\right)^{n} e^{-\zeta_{2}^{\prime}(0)} \\
& \leq(1+o(1)) \frac{2 \log n}{k n \log k}\left(\frac{(k-1)^{k-1}}{\left(k^{2}-2 k\right)^{k / 2-1}}\right)^{n} .
\end{aligned}
$$

Theorem 4 is proved.

Proof of Theorem 5: 
For a graph with girth (the length of the smallest cycle) $g$, we can take $\beta=\lfloor g / 2\rfloor$ and we have

$$
\begin{aligned}
\zeta_{1}(s)= & \frac{1}{\Gamma(s)} \int_{0}^{\infty} t^{s-1} e^{-t}\left(\sum_{j \geq 0} r_{j}^{\prime} \frac{(t / k)^{j}}{j !}-e^{t}\right) d t \\
= & \frac{1}{\Gamma(s)} \int_{0}^{\infty} t^{s-1} e^{-t}\left(-\sum_{j=0}^{g} \frac{(t / k)^{j}}{j !}\right) d t \\
& +\frac{1}{\Gamma(s)} \int_{0}^{\infty} t^{s-1} e^{-t}\left(\sum_{j>g}\left(r_{j}^{\prime} \frac{(t / k)^{j}}{j !}-\frac{(t / k)^{j}}{j !}\right)\right) d t \\
\zeta_{1}^{\prime}(0) \leq-\sum_{j=1}^{g-1} \frac{1}{j}+\zeta_{2}^{\prime}(0) & \leq-\log g+\zeta_{2}^{\prime}(0)
\end{aligned}
$$

where here we will need to use some known results on random $k$-regular graphs. Erdős and Sachs [8] proved that with positive probability, say at least $1 / 2$, there is a $k$-regular graph on $n$ vertices having girth $g$ satisfying

$$
g=(1+o(1)) \frac{\log n}{\log k}
$$

as $n$ approaches infinity. Friedman [10] showed that with probability approaches 1 , the expected number of irreducible walks $c_{j}(v)$ rooted at a vertex $v$ of length $j$, for $k \geq 8$, is

$$
E\left(c_{j}(v)\right)=k(k-1)^{j-1}\left(\frac{1}{n}+\operatorname{Err}_{n, j}\right)
$$

where

$$
E r r_{n, j}=O\left((c k j)^{c}\left(\frac{j^{2 \sqrt{k}}}{n^{1+\sqrt{k-1} / 2}}+\frac{1}{k^{j / 2}}\right)\right) .
$$

We note that in the original paper of Friedman, only the case for even $k$ was treated. However, the argument of counting irreducible "words" made of letters can be extended to counting walks on the $k$-trees for odd $k$ in a similar way.

The expected number of closed walks of length $j$ satisfies (see [10])

$$
k^{j}\left(1+(n-1) p_{j, 0}+\sum_{s=1}^{j} n p_{j, s} E r r_{n, s}\right)
$$

where $p_{j, s}$ is the probability that a random walk of length $j$ reduces to an irreducible walk of size $s$. Hence, the number $r_{j}^{\prime}$ of not totally reducible walks of length $j$ satisfies

$$
E\left(\frac{r_{j}^{\prime}}{k^{j}}\right)=1-p_{j, 0}+\sum_{s=1}^{j} n p_{j, s} E r r_{n, s} .
$$


Since $p_{j, s} \leq 2^{j} k^{-j+s}$, we have

$$
\begin{aligned}
\zeta_{2}(s) & =\frac{1}{\Gamma(s)} \int_{0}^{\infty} t^{s-1} e^{-t}\left(\sum_{j \geq 2 \beta}\left(r_{j}^{\prime} \frac{(t / k)^{j}}{j !}-\frac{(t / k)^{j}}{j !}\right)\right) \\
& \leq \frac{1}{\Gamma(s)} \int_{0}^{\infty} t^{s-1} e^{-t}\left(\sum_{j \geq 2 \beta} \frac{(t / k)^{j}}{j !} 2^{j} \sum_{s=0}^{j} k^{-j+s}(c k s)^{c}\left(\frac{s^{2 \sqrt{k}} 2^{s}}{n^{\sqrt{k-1} / 2}}+\frac{1}{(k-1)^{s / 2}}\right)\right) .
\end{aligned}
$$

Therefore,

$$
\begin{aligned}
\zeta_{2}^{\prime}(0) & \leq \sum_{j \geq g} \frac{1}{j} \sum_{s=1}^{j} 2^{j} k^{-j+s}(c k s)^{c}\left(\frac{s^{2 \sqrt{k}} 2^{s}}{n^{\sqrt{k-1} / 2}}+\frac{1}{(k-1)^{s / 2}}\right) \\
& =o(1) .
\end{aligned}
$$

Using (10) and combining the preceding bounds, we have

$$
\begin{aligned}
\tau(G) & =\frac{1}{k n}\left(\frac{(k-1)^{k-1}}{\left(k^{2}-2 k\right)^{k / 2-1}}\right)^{n} e^{-\zeta_{1}^{\prime}(0)} \\
& \geq \frac{g}{k n}\left(\frac{(k-1)^{k-1}}{\left(k^{2}-2 k\right)^{k / 2-1}}\right)^{n} e^{-\zeta_{2}^{\prime}(0)} \\
& \geq(1+o(1)) \frac{\log n}{k n \log k}\left(\frac{(k-1)^{k-1}}{\left(k^{2}-2 k\right)^{k / 2-1}}\right)^{n} .
\end{aligned}
$$

This completes the proof of Theorem 5 .

\section{References}

[1] C. T. Benson, Minimal regular graphs of girth eight and twelve, Canad. J. Math. 18 (1996), 1091-1094.

[2] N. Biggs, Algebraic Graph Theory, Cambridge University Press, 1993.

[3] R. Brooks, The spectral geometry of $k$-regular graphs, Journal D'analyse Mathématrique, 57 (1991) 120-151.

[4] J. Cheeger and S.-T. Yau, A lower bound for the heat kernel, Communications on Pure and Applied Mathematics XXXIV (1981), 465-480.

[5] D. M. Cvetković, M. Doob and H. Sachs, Spectra of graphs, Academic Press, New York, 1980.

[6] F. R. K. Chung and S.-T. Yau, A combinatorial trace formula, Tsing Hua Lectures on Geometry and Analysis, (ed. S.-T. Yau), International Press, Cambridge, Massachusetts, 1997, 107-116. 
THE ELECTRONiC JoURnal of COMBInAtorics 6 (1999), \#R12

[7] F. R. K. Chung, Spectral Graph Theory, CBMS Lecture Notes, 1997 , AMS Publication.

[8] P. Erdős and H. Sachs, Reguläre Graphen gegenebener Teillenweite mit Minimaler Knotenzahl, Wiss. Z. Univ. Halle - Wittenberg, Math. Nat. R. 12 (1963), 251-258.

[9] W. Feit and G. Higman, The non-existence of certain generalized polygons, J. Algebra 1 (1964), 114-131.

[10] J. Friedman, On the second eigenvalues and random walks in random $d$-regular graphs, Combinatorica 11 (1991), 331-362.

[11] A. K. Kelmans, On properties of the characteristic polynomial of a graph, Kibernetiku-Na Sluzbu Kommunizmu 4 Gosenergoizdat, Moscow, 1967.

[12] C. D. Godsil and B. D. McKay, Feasibility conditions for the existence of walkregular graphs, Linear Algebra Appl., 30 (1980), 51-61.

[13] F. Kirchhoff, Über die Auflösung der Gleichungen, auf welche man bei der Untersuchung der linearen Verteilung galvanischer Ströme geführt wird, Ann. Phys. chem. 72 (1847) 497-508.

[14] B. D. McKay, Backtrack programming and the graph isomorphism problem, M. Sc. Thesis, University of Melbourne, 1976.

[15] B. D. McKay, The expected eigenvalue distribution of a large regular graph, Linear Algebra and its Applications 40 (1981), 203-216.

[16] B. D. McKay, Spanning trees in regular graphs, Europ. J. Combinatorics 4 (1983), 149-160.

[17] Peter Sarnak, Some Applications of Modular Forms, Cambridge University Press, New York, 1990.

[18] Richard M. Schoen and S.-T. Yau, Lectures on Differential Geometry, International Press, Cambridge, Massachusetts, 1994.

[19] R. R. Singleton, On minimal graphs of maximum even girth, J. Comb. Th 1 (1966), 306-322. 